\title{
Efeitos de três modalidades de atividade física na capacidade funcional de idosos
}

CDD. 20.ed. 613.7

796.018

\author{
Deisy Terumi UENO* \\ Sebastião GOBBI* \\ Camila Vieira Ligo TEIXEIRA* \\ Émerson SEBASTIÃO* \\ Alexandre Konig Garcia PRADO** \\ J osé Luiz Riani COSTA* \\ Lilian Teresa Bucken GOBBI* \\ *Instituto de Biociên- \\ cias, Universidade \\ Estadual Paulista - Rio \\ Claro. \\ **Department of Kine- \\ sioloy and Community \\ Health, University of \\ Illinois at Urbana - \\ Champaign - USA.
}

\section{Resumo}

0 objetivo do presente estudo foi analisar e comparar os efeitos de três modalidades de atividade física: atividade física geral, dança e musculação, na capacidade funcional de idosos. A amostra foi composta por 94 idosos com idade média de 64,06 \pm 7,38 anos, que participavam do PROFIT. Para a avaliação foi utilizada a bateria de testes da AAHPERD, da qual se obtém os niveis de flexibilidade, coordenação, agilidade e equilibrio dinâmico, resistência de força e resistência aeróbia geral, antes e após quatro meses de intervenção. Os resultados apontaram de uma maneira geral uma manutenção nos níveis dos componentes da capacidade funcional. Os participantes do grupo atividade física geral obtiveram melhora dos resultados nos componentes: resistência de força, agilidade e equilibrio dinâmico. Pode-se concluir que a prática de atividade física regular e sistematizada, independentemente da modalidade, pode ter influência positiva no desempenho funcional de idosos, podendo preservar sua independência e autonomia.

Unitermos: Envelhecimento; Exercício físico; Aptidão funcional.

\section{Introdução}

Nos últimos anos observa-se um crescimento significativo da população idosa devido, dentre outros fatores, aos avanços tecnológicos e da saúde bem como as baixas taxas de natalidade. Segundo o Instituto Brasileiro de Geografia e Estatística, em 2008 o Brasil possuía cerca 21 milhôes de idosos e, estima-se que até o ano de 2050 existirão aproximadamente 64 milhões de idosos, correspondendo a cerca de $30 \%$ da população brasileira (IBGE, 2008).

O processo de envelhecimento é caracterizado por alterações biológicas, funcionais, sociais e intelectuais que ocorrem de forma natural, dinâmica, progressiva e irreversível (JoRDÃo NetTO, 1997; SANTOS, 2000; ZAGO \& GoBBI, 2003). Muitas vezes, tal processo associado ao baixo nível de atividade física favorece o desenvolvimento de doenças crônicas, outras incapacidades e, por consequência o declínio da capacidade funcional (CF), que podem levar o idoso a um quadro de dependência (COELHO, Quadros Júnior \& GobBi, 2008).

Dessa forma, na relação entre envelhecimento, saúde e aptidão física, o conceito de CF torna-se um dos fatores mais relevantes na análise dos efeitos do envelhecimento, refletindo esse processo, melhor que a idade cronológica (Clark, 1989; Coelho, QuAdRos JúNIOR \& GobBI, 2008; MATSUdo, 2001).

Evidências científicas sugerem que a prática de atividade física é um importante instrumento na recuperação, manutenção e promoção da saúde e consequentemente da qualidade de vida (CHODzJo-ZajKO, Proctor, Fiatarone Singh, Minson, Nigg, Salem \& SKINNER, 2009). Tal prática vem sendo apontada como uma das formas de postergar muitos dos declínios físicos, psicológicos e sociais ocasionados pelo envelhecimento, preservando assim, a CF, independência e autonomia dos idosos (Benedetti, Mazo, 
Gobbi, Amorim, Gobbi, Ferreira \& Hoefelmann, 2007; Gobbi, 1997; Matsudo, Matsudo, Araújo, Andrade, Andrade, Oliveira \& Bragion, 2002).

A CF, segundo Clark (1989) é definida como a capacidade de realizar as atividades ordinárias e inesperadas do dia a dia, de forma segura e eficaz e sem cansaço excessivo. Tal capacidade é composta por seis componentes, quais sejam: resistência de força, agilidade, equilíbrio, flexibilidade, coordenação e resistência aeróbia. Assim, o nível dos componentes da CF reflete diretamente no sucesso ou fracasso durante a realização das atividades de vida diária.

Diferentes protocolos/programas de atividade física têm sido desenvolvidos com idosos, com o intuito de conhecer seus efeitos sobre a CF ou de seus componentes (Dias, GURJĀo \& MARUCCI, 2006; Sebastiâo, Hamanaka, Gobbi \& Gobbi,

\section{Métodos}

O presente estudo apresenta delineamento de pesquisa retroativa, o qual se baseou em análise do banco de dados do Programa de Atividade Física para Terceira Idade (PROFIT). O referido programa é desenvolvido no Departamento de Educação Física - Instituto de Biociências - UNESP - Campus de Rio Claro. O PROFIT é um projeto de extensão que oferece atividade física sistematizada, quais sejam: a) Musculação; b) Atividade Física Geral; c) Dança; d) Alongamento; e) "Square Stepping Exercise" (Shiguematsu \& OKURA, 2006).

O referido programa é desenvolvido há mais de 20 anos e, para efeito do presente manuscrito, optou-se por desenvolver a análise dos dados somente dentre as três atividades mais antigas do projeto (Musculação, Dança e Atividade Física Geral). Os dados analisados são referentes às duas avaliações realizadas pelos idosos, após período de férias, antes e após 16 semanas do segundo semestre de 2009 (agosto e dezembro).

\section{Sujeitos}

Participaram do estudo 94 idosos, 14 homens e 80 mulheres, com idade média de 64,06 \pm 7,38 anos, aparentemente saudáveis, residentes na cidade de Rio Claro/SP, que participavam, em média, a 4,17 \pm 4,56 anos do PROFIT. Foram utilizados os resultados das avaliaçôes dos seguintes grupos: Grupo Atividade Física Geral (GAFG) n = 49, Grupo Dança (GD) n = 16, e Grupo Musculação (GM) n = 29. Para os critérios de inclusão adotou-se: a) ter mínimo de $75 \%$ de presença
2008; Silva, Santos Filho \& Gobbi, 2006; ZaGO, Polastri, Villar, Silva \& GobBi, 2000). Dentre eles, o treinamento com pesos, a dança e a atividade física geral tem sido constantemente investigados (Coelho, Quadros Júnior \& GobBi, 2008; Dias et al., 2006; SEBASTIĀO et al., 2008; SILVA et al., 2006; ZAGO et al., 2000). No entanto, tais atividades têm sido avaliadas de maneira isolada, cabendo investigar, dentre estas, qual poderia trazer maiores benefícios à população idosa, no que se refere à CF, uma vez que cada modalidade é composta por atividades/protocolos, com princípios que as diferenciam bastante uma da outra.

Dessa maneira, o presente estudo procurou analisar e comparar o efeito de três diferentes modalidades de atividade física sistematizada: dança, atividade física geral e musculação, na CF de idosos.

durante o período que compreendeu a intervenção (controle realizado por meio de lista de presença); b) ter completado todos os testes que compõe a bateria de testes, anterior ao periodo de treinamento e posterior ao período de treinamento de quatro meses (pré e pós); c) estar participando da modalidade por um período não inferior a seis meses. É importante salientar que dados de quatro sujeitos do GAFG, cinco do GD e 12 do GM foram excluídos por não atenderem aos critérios de inclusão. Todos os participantes assinaram um termo de consentimento de participação no estudo, permitindo publicação dos dados obtidos nas avaliações.

\section{Protocolo de avaliação}

O PROFIT adota como procedimento padrão, desde o seu surgimento, a avaliação dos participantes por meio da bateria de testes motores da American Alliance for Health, Physical Education, Recreation and Dance - AAHPERD (Osness, Clark, Hoeger, RaAB \& WisWELL, 1990). A referida bateria tem estudo de validade e confiabilidade proposto por diversos autores em diferentes métodos (BRAVO, GAUTHIER, ROY, TESSIER, Gaulin, Dubois \& Peloquin, 1994; Mobily \& Mobily 1997; YAGUCHI \& FURUTANI 1998; ZAGO et al., 2000). A opção pela utilização da referida bateria deve-se as seguintes circunstâncias: a) praticidade, pois permite avaliar grande número de idosos em pouco tempo e com o mínimo de equipamento; b) especificamente delineada para idosos; c) minimiza riscos devido à natureza dos 
testes; e d) as tarefas motoras envolvidas são similares às atividades realizadas no cotidiano.

A bateria da AAHPERD é composta por cinco testes motores, quais sejam:

a) Flexibilidade (FLEX) - o participante sentado no solo, com as pernas estendidas, se posiciona sobre a marca zero de uma fita métrica afixada no solo. À marca de $63,5 \mathrm{~cm}$ de distância em relação à marca zero da fita, é afixada perpendicularmente uma fita adesiva que indica a posição dos calcanhares que devem estar 15,2 cm equidistantes do centro da fita métrica. Então, com as mãos uma sobre a outra, o participante é orientado a deslizá-las vagarosamente sobre a fita métrica tão distante quanto pode, permanecendo na posição final no mínimo por dois segundos. São oferecidas duas tentativas de prática, seguidas de duas tentativas de teste. O resultado final é dado pela melhor das duas tentativas anotadas;

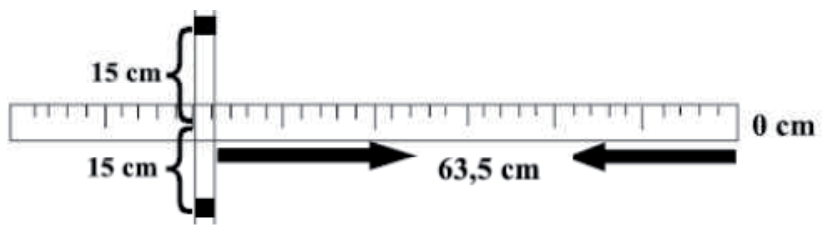

FIGURA 1 - Ilustração gráfica do teste de flexibilidade (ZAGO \& GoBBI, 2003; adaptada de OsNESs et al., 1990).

b) Coordenação Óculo-Manual (COO) - o participante deve permanecer sentado em uma cadeira, de frente para uma mesa na qual está afixada uma fita adesiva de $76,2 \mathrm{~cm}$ de comprimento, contendo seis marcaçôes equidistantes entre si. Com sua mão dominante e o polegar voltado para cima, a base de apoio de três latas deve ser invertida, de forma que a lata 1 que está na posição 1 seja colocada na posição 2; a lata 2 da posição 3 para a posição 4 e; a lata 3 da posição 5 para a 6 . Sem perda de tempo, o avaliado, estando agora com o polegar apontado para baixo, apanha a lata 1 e inverte novamente sua base, recolocando-a na posição $1 \mathrm{e}$, da mesma forma, procede colocando a lata 2 na posição 3 e a lata 3 na posição 5 , completando assim um circuito. O participante deve completar dois circuitos. São concedidas duas tentativas de prática, e considerado como resultado final o menor dos tempos obtidos;

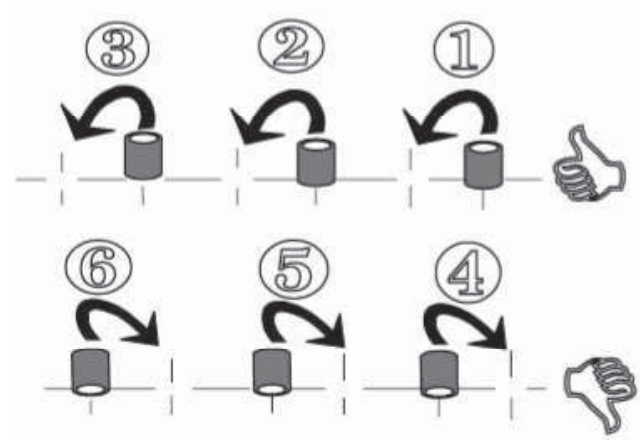

FIGURA 2 - Ilustração gráfica do teste de coordenação óculo-manual (ZAGO \& GoBBI, 2003; adaptada de OsNESS et al., 1990).

c) Agilidade e Equilíbrio Dinâmico (AGILEQ) - Inicialmente sentado numa cadeira, o avaliado deve deslocar-se o mais rápido possível para a direita e circundar um cone que está posicionado a $1,50 \mathrm{~m}$ para trás e 1,80 m para o lado da cadeira, retornando e sentando-se. Imediatamente o participante deve levantar-se, mover-se para a esquerda e circundar o segundo cone posicionado a mesma distância do primeiro, retornando para a cadeira e sentando-se novamente, completando assim um ciclo. Cada tentativa corresponde à execução de dois ciclos completos. São realizadas duas tentativas e o melhor tempo (o menor) é anotado em segundos como o resultado final; 


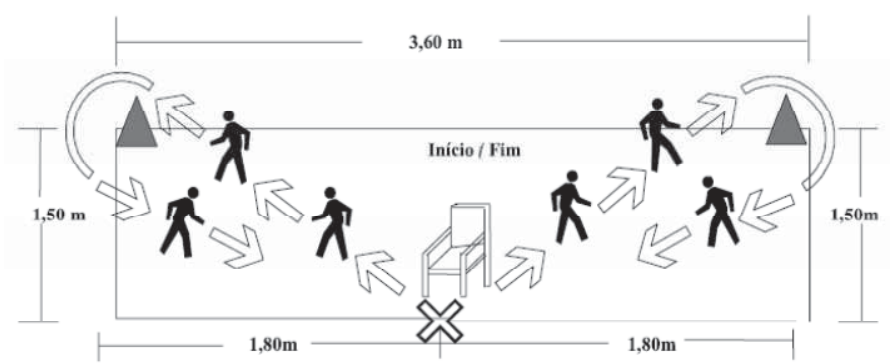

FIGURA 3 - Ilustração gráfica do teste de agilidade e equilíbrio dinâmico (ZAGO \& GoBBI, 2003; adaptada de OsNEss et al., 1990).

d) Resistência de força para membros superiores (RESISFOR) - O participante deve permanecer sentado em uma cadeira sem braços, apoiando as costas no encosto da cadeira, com o tronco ereto, olhando para frente, com o braço não dominante apoiado sobre a coxa e com a planta dos pés completamente apoiadas no solo. Então, o participante é orientado a realizar com o braço dominante, o maior número de flexões de cotovelo possíveis dentro de 30 segundos, segurando um halter (1,84 kg para as mulheres e 3,63 kg para homens). Inicialmente o halter deve estar paralelamente ao solo com uma de suas extremidades voltadas para frente. São contabilizadas somente as repetições realizadas com extensão e flexão completa do cotovelo;

e) Resistência Aeróbia Geral (RAG) - O participante é orientado a caminhar (sem correr) 804,67 metros, numa pista de atletismo de $400 \mathrm{~m}$, o mais rápido possível. É oferecida apenas uma tentativa por participante e o tempo gasto para realizar tal tarefa é anotado em minutos e segundos e, posteriormente reduzidos a segundos.

Importante destacar que todos os testes foram aplicados no mesmo espaço físico e horário para todos os grupos.

\section{Rotina de exercícios}

No PROFIT, as sessões de todas as atividades ocorrem três vezes por semana com duração de 45 a 60 minutos cada. Todas as atividades apresentam em sua estrutura, aquecimento, parte principal e volta à calma (relaxamento). As sessōes de musculação eram realizadas em dias não consecutivos (segundas, quartas e sextas-feiras) com duração de 45 minutos nos quais eram realizados oito exercícios alternados por segmentos corporais, sendo três séries para cada exercício. $\mathrm{O}$ intervalo de recuperação entre as séries foi de aproximadamente um minuto, e as participantes foram constantemente instruídas a realizarem cada repetição em aproximadamente três segundos. A carga foi determinada por meio do teste de 15 repetições máximas (15 RM), com intensidade de treinamento variando entre 10-15 RM. As sessões de Dança e Atividade Física Geral ocorreram três vezes por semana, em dias consecutivos (terças, quartas e quintas-feiras), com duração de 60 minutos, com intensidade moderada controlada pela percepção do professor responsável. A primeira consiste na prática de sequências rítmicas coreografadas, a qual é elaborada com sequências de passos que formam uma coreografia, sendo realizado com um mínimo tempo de intervalo entre as repetições e as sequências; a segunda consiste na execução de atividades diversificadas, como por exemplo, esporte adaptado, atividade lúdica, exercícios calistênicos, caminhada, circuito e alongamento.

Cabe salientar, que todas as sessôes de atividades eram acompanhadas por profissionais de Educação Física e com a colaboração de alunos/estagiários, do curso de graduação em Educação Física da UNESP - campus Rio Claro.

\section{Procedimento estatístico}

Para caracterização da amostra foi utilizada análise descritiva (média e desvio-padrão). Após verificada a normalidade da distribuição dos dados pelo teste de Kolmogorov Smirnov, para as medidas inter e intra grupos foi utilizada a análise de variância (ANOVA $3 \times 2$ ), para dois fatores (treino e tempo). Os efeitos principais analisados pela ANOVA $3 \times 2$ foram os três níveis da variável independente Grupo (Atividade Física Geral, Dança e Musculação) e os dois níveis da variável independente Tempo (momentos antes e após intervenção - Pré e Pós), além da interação entre essas duas variáveis (Grupo vs Tempo). Nos componentes nos quais as condições iniciais dos grupos eram diferentes, a análise de covariância (ANCOVA) para medidas repetidas foi empregada, com os valores pré-treinamento sendo utilizados como covariáveis. O teste "post hoc" LSD foi utilizado para a identificação das diferenças específicas nas variáveis em que os valores foram inferiores ao nível de significância estabelecido $(\mathrm{p} \leq 0,05)$. 


\section{Resultados}

Os valores de caracterização da amostra estão apresentados na TABELA 1. A Anova apontou diferença significativa $(F=3,90, p=0,02)$ entre os grupos apenas para a variável idade. O "post hoc" LSD mostrou que o GD era o grupo mais novo quando comparado aos outros grupos.

Os resultados das avaliações nos momentos PRÉ e PÓS, bem como os valores de F e p obtidos na análise de variância são apresentados na TABELA 2. O "post hoc" LSD apontou que no momento pré, houve diferença significativa entre os grupos nos testes de AGILEQ $(\mathrm{F}=6,46, \mathrm{p}=0,00)$, RESISFOR $(\mathrm{F}=9,32, \mathrm{p}=0,00)$ e RAG $(\mathrm{F}=3,75, \mathrm{p}=0,02)$, sendo que $\mathrm{GD}$ apresentou o maior valor médio no teste AGILEQ comparado ao GAFG $(\mathrm{p}=0,01)$ e
GM ( $p=0,00)$ e no teste de RESISFOR (GAFG: $p=0,01, G M: p=0,00)$. Para o teste de RAG, o GAFG apresentou os piores resultados comparado ao GD $(\mathrm{p}=0,05)$ e GM ( $\mathrm{p}=0,05)$.

Analisando o comportamento de cada grupo nos momentos pré e pós, apenas o GAFG apresentou melhora nos testes AGILEQ ( $\mathrm{p}=0,05)$ e RESISFOR $(\mathrm{p}=0,01)$.

Assim, a ANCOVA foi empregada para os testes AGILEQ, RESISFOR e RAG, tendo os valores prétreinamento como covariantes. Porém as médias dos grupos para estes componentes não foram significativamente diferentes quando controlada pela covariante, AGILEQ $(\mathrm{F}=1,29, \mathrm{p}=0,28)$, RESISFOR $(\mathrm{F}=$ $0,13, \mathrm{p}=0,87)$ e $\mathrm{RAG}(\mathrm{F}=1,90, \mathrm{p}=0,15)$.

TABELA 1 - Valores de idade, tempo de participação no PROFIT, estatura e massa corporal da amostra apresentados em média e desvio padrão, e valores de $\mathrm{F}$ e p referentes à análise de variância.

\begin{tabular}{lccccc}
\hline Variável & GAFG & GD & GM & F & p \\
\hline Idade (anos) & $64,6 \pm 7,57^{*}$ & $59,6 \pm 7,40$ & $65,6 \pm 6,24 *$ & 3,902 & 0,02 \\
Participação (anos) & $3,8 \pm 4,61$ & $4,50 \pm 3,86$ & $4,54 \pm 4,92$ & 0,273 & 0,76 \\
Estatura (cm) & $158,57 \pm 7,24$ & $157,00 \pm 6,24$ & $161,69 \pm 7,25$ & 2,749 & 0,07 \\
Massa Corporal (kg) & $71,02 \pm 12,55$ & $70,14 \pm 11,30$ & $75,29 \pm 12,79$ & 0,431 & 0,65 \\
\hline
\end{tabular}

GAFG = Grupo Atividade Física Geral; GD = Grupo Dança; $\mathrm{GM}=$ Grupo Musculação. $p \leq 0,05$.

* diferença significativa em relação ao GD.

TABELA 2 - Valores obtidosnas avaliações dos componentes dacapacidadefuncional apresentados em médiaedesvio padrão nos momentos Pré e Pós quatro meses de treinamento e valores de F e p para cada componente.

\begin{tabular}{|c|c|c|c|c|c|c|}
\hline & GAFG & GD & GM & Efeitos & $\mathbf{F}$ & $\mathbf{P}$ \\
\hline \multicolumn{4}{|c|}{ Flexibilidade (centímetros) } & Grupo & 1,42 & 0,24 \\
\hline Pré & $59,6 \pm 11,4$ & $59,1 \pm 12,6$ & $55,5 \pm 12,0$ & Momento & 1,23 & 0,27 \\
\hline Pós & $59,4 \pm 12,4$ & $62,5 \pm 10,9$ & $56,6 \pm 11,3$ & Grupo*momento & 0,59 & 0,55 \\
\hline \multicolumn{4}{|c|}{ Coordenação Óculo Manual (segundos) } & Grupo & 0,85 & 0,43 \\
\hline Pré & $9,6 \pm 1,8$ & $8,8 \pm 0,9$ & $9,2 \pm 1,0$ & Momento & 3,89 & 0,52 \\
\hline Pós & $10,1 \pm 2,7$ & $10,1 \pm 5,2$ & $9,5 \pm 1,1$ & Grupo*momento & 0,59 & 0,55 \\
\hline \multicolumn{4}{|c|}{ Agilidade e Equilíbrio Dinâmico (segundos) } & Grupo & 6,97 & $0,00^{*}$ \\
\hline Pré & $21,3 \pm 3,3$ & $18,4 \pm 2,2$ & $21,0 \pm 2,1$ & Momento & 2,07 & 0,15 \\
\hline Pós & $20,2 \pm 3,8$ & $18,2 \pm 2,1$ & $20,6 \pm 2,0$ & Grupo*momento & 0,61 & 0,55 \\
\hline \multicolumn{4}{|c|}{ Resistência de Força (repetiçóes) } & Grupo & 7,57 & $0,00^{*}$ \\
\hline Pré & $32,2 \pm 5,6$ & $38,9 \pm 5,4$ & $34,1 \pm 5,0$ & Momento & 1,05 & 0,30 \\
\hline Pós & $34,2 \pm 5,0$ & $38,1 \pm 4,7$ & $34,8 \pm 5,3$ & Grupo*momento & 1,73 & 0,18 \\
\hline \multicolumn{4}{|c|}{ Resistência Aeróbia Geral (segundos) } & Grupo & 5,60 & $0,00^{*}$ \\
\hline Pré & $504,0 \pm 73,9$ & $461,5 \pm 46,7$ & $474,9 \pm 43,8$ & Momento & 0,91 & 0,34 \\
\hline Pós & $499,1 \pm 62,0$ & $455,0 \pm 41,9$ & $468,7 \pm 42,2$ & Grupo*momento & 0,01 & 0,10 \\
\hline
\end{tabular}

GAFG = Grupo Atividade Física Geral; $\mathrm{GD}=$ Grupo Dança; GM= Grupo Musculação. ${ }^{*} p \leq 0,05$ 


\section{Discussão}

O objetivo desse estudo foi analisar e comparar o efeito de três diferentes modalidades de atividade física sistematizada: atividade física geral, dança e musculação, na CF de idosos.

Ao comparar os grupos no momento pré, é possível verificar que o GD apresentava resultados melhores que os outros grupos nos testes de AGILEQ e RESISFOR, o que pode ser explicado pelo fato dos idosos apresentarem menor média de idade (Matsudo, Matsudo, Barros Neto \& Araújo, 2003). Além disso, o fato desses idosos serem previamente treinados nessa modalidade pode ter influenciado nestes resultados, uma vez que a dança exige movimentos com rápida mudança de direção e/ou de altura do centro de gravidade, estimulando assim a agilidade e o equilíbrio dinâmico. A dança também apresenta característica de maior sustentação de parte do peso corporal e maior repetição de movimentos comparada às outras modalidades, o que poderia explicar o melhor desempenho no teste de RESISFOR (COELHO, QUADROS JúNIOR \& GobBI, 2008; SebastiÃo et al., 2008).

O GAFG apresentou o pior resultado no teste de RAG, indicando que apesar dos idosos serem treinados e do protocolo de treinamento ser diversificado, o mesmo não teria sido suficiente para melhorar a RAG dos idosos participantes desta modalidade, sendo necessária outra estratégia para provocar a melhora deste componente. No entanto, quando analisamos o comportamento de cada grupo entre as avaliações, melhoras significativas foram evidenciadas nos resultados dos testes de AGILEQ e de RESISFOR de membros superiores para o GAFG.

Nossos achados corroboram o estudo de CARVALHAIS (2004) que encontrou melhoras significativas no componente agilidade e equilíbrio dinâmico após oito meses de um programa de atividade física generalizada em idosos que participavam a três anos do programa em média. MiYasiKe-DA-Silva, VILLAR, ZAGO, POlastri e GobBi (2002) analisaram em quatro avaliações, sendo duas em cada semestre, o nível de agilidade de 26 indivíduos, com idade média de 58 anos que realizavam atividade física generalizada durante um ano. Os autores observaram melhora significativa na segunda e terceira avaliações e posterior manutenção para este componente após um ano de treinamento. Esses resultados vão contra os nossos achados que demonstraram uma melhora significativa entre as avaliações realizadas no início e final do segundo semestre.
Em relação à melhora para o componente RESISFOR para o GAFG observada em nosso estudo, tais resultados corroboram os achados de ZAGO et al. (2000), que encontraram melhora significativa no nível de força em idosos participantes de um programa de atividade física geral, com duração de nove meses. Embora ambos os estudos tenham observado melhora no componente RESISFOR em indivíduos ativos, nosso estudo demonstra que até mesmo em menores períodos de treinamento (quatro meses), resultados positivos podem ser observados.

Um estudo conduzido por Pauli, Souza, ZAGO e Goвbi (2009), verificou os efeitos de 12 anos de prática de atividade física em programa supervisionado sobre a aptidão funcional de idosos. Os autores encontraram melhora significativa nos componentes COO, FLEX, AGILEQ, e manutenção dos componentes RESISFOR e RAG. Porém, o referido autor realizou apenas duas avaliações, antes e após 12 anos, o que não permite verificar a partir de qual momento houve melhora ou manutenção dos níveis iniciais. CAIXETA Junior e BARros (2009) encontraram apenas manutenção nos componentes da CF de idosos que participavam de programas de atividade física na cidade de Patos de Minas, Estado de Minas Gerais. No entanto, os autores realizaram duas avaliaçóes, sendo a segunda após um mês da primeira, o que parece ser pouco tempo para observar melhoras nos níveis iniciais dos componentes da CF.

Os GD e GM não apresentaram melhoras entre as avaliações, o que pode ser explicado pelo fato dos idosos já terem atingido os níveis máximos desses componentes, visto que já eram treinados à pelo menos seis meses. Porém cabe ressaltar, que o processo de envelhecimento é caracterizado por uma diminuição progressiva dos componentes $\mathrm{da} \mathrm{CF}$, e o fato do treinamento manter os níveis dos componentes é de grande importância, visto que a sobrecarga do exercício já estaria sendo imposta pelo próprio avançar da idade (Gobbi, Villar \& ZaGo, 2005).

O GM apresentou uma manutenção nos resultados do teste RESISFOR, apesar do protocolo de treinamento ser mais específico para o desenvolvimento deste componente. Uma possível explicação é que as sessões de treinamento, que consistiam na realização de três séries de 12 a 15 repetições com aproximadamente $70 \%$ da carga máxima, podem não ter proporcionado melhora específica para o componente RESISFOR. Possíveis alterações causadas por essa rotina de treinamento podem não ter 
sido detectadas no teste de RESISFOR, que consiste na execução do maior número de repetiçõos possíveis em apenas 30 segundos. Vital, Patatas, Tavares, Coelho, Costa, Nunes e Puga (2011) citam também que, segundo o princípio da especificidade do treinamento, esse teste pode não ser específico para avaliar as alteraçóes decorrentes de um treinamento resistido, da mesma forma que o teste de 1 RM.

É importante destacar algumas limitações, que podem ter interferido nos resultados do presente estudo. Os participantes já eram treinados e possuíam bons resultados no momento inicial, podendo já ter alcançado o seu limite biológico, que, quando alcançado, impossibilita avanços nos níveis dos componentes da CF. Os idosos também já possuíam uma familiaridade com os testes, podendo facilitar a realização dos testes e influenciar diretamente nos resultados.

Os participantes do GD eram mais novos, o que poderia ser explicado devido à menor procura de pessoas mais idosas para esta modalidade, talvez por receio destas pessoas em realizar movimentos mais complexos, como giros, saltos, sequências de movimentos, entre outros, pois a modalidade de dança do presente estudo é caracterizada por ritmos diferentes aos geralmente desenvolvidos em bailes de terceira idade.

Os dados obtidos são apenas uma parcela das avaliações dos idosos que já participavam em média há quatro anos, não sendo possível identificar o ganho obtido pelos mesmos desde o inicio da participação no programa.

A falta de um grupo controle, no presente estudo, não permite inferir qual modalidade seria melhor, não sendo possível ter certeza de que os resultados encontrados são de fato em resposta ao período de intervenção. Porém, é possível afirmar que as três modalidades surtem efeito positivo na CF de idosos, visto que a manutenção dos níveis de CF está associada à manutenção da independência e autonomia para a realização das tarefas do dia a dia. Assim, recomendam-se programas de atividade física voltados para idosos que incluam modalidades variadas, de forma a desenvolver por meio de estímulos diversificados a melhora e/ou manutenção dos componentes da CF em idosos.

\title{
Conclusão
}

Podemos concluir que idosos previamente treinados a quatro anos em média, que participaram de um protocolo de treinamento de atividade física geral, obtiveram melhoras significativas nos componente agilidade e equilíbrio dinâmico, e resistência de força, o que não ocorreu nos grupos de dança e musculação. Os demais componentes analisados apresentaram uma manutenção após quatro meses de intervenção, sendo possível inferir que a prática regular de atividade física orientada e sistematizada, independente da modalidade, é um instrumento eficaz para a manutenção dos componentes da CF de idosos treinados. A simples manutenção dos componentes da CF representa um impacto bastante positivo, visto que a curva de declínio da CF é mais acentuada em idosos.

\begin{abstract}
Effects of three methods of physical activity in functional ability of elderly

The aim of this study was to analyze and compare the effects of three types of physical activity: general physical activity, dance and weight training, in functional capacity in elderly. The sample included 94 seniors with a mean age of $64.06 \pm 7.38$ years, who participated in PROFIT. For the evaluation we used the battery of test from AAHPERD, which checks the levels of flexibility, coordination, agility and dynamic balance, strength endurance and general endurance before and after four months of intervention. The results indicated a maintained in the levels of functional components. The participants general physical activity group had better results on the components: strength endurance, agility and dynamic balance. It can be concluded that the practice of regular physical activity and systematized, regardless of the type may have a positive influence on the functional performance of elderly and can preserve their independence and autonomy.
\end{abstract}

UnITERMs: Elderly; Physical activity; Functional capacity. 


\section{Resumen}

Efectos de tres modalidades de actividad física en la capacidad funcional de adultos mayores

El objetivo de este estudio fue analizar y comparar los efectos de actividad física generalizada, danza y musculación en la capacidad funcional de adultos mayores. La muestra consistió en 94 adultos mayores con promedio de edad de 64,06 \pm 7,38 años, participantes en el PROFIT. Para la evaluación se utilizó de la batería de testes de la AAHPERD, la cual se puede obtener los niveles de flexibilidad, coordinación, agilidad y equilibrio dinámico, resistencia de fuerza y resistencia general, antes y después de cuatro meses de intervención. Los resultados mostraron una manutención en los niveles de los componentes funcionales. Lo grupo de actividad física general tuvieron mejores resultados en la resistencia de fuerza, agilidad y equilibrio dinámico. Se conclui que la práctica de actividad física regular y sistematizada, independiente dela modalidad, puede tener una influencia positiva en el desempeño funcional de los adultos mayores y pueden preservar su independencia y autonomía.

Palabras Clave: Envejecimiento; Ejercicio físico; Aptitud funcional.

\section{Referências}

BENEDETTI, T.R.B.; MAZO, G.Z.; GOBBI, S.; AMORIM, M.; GOBBI, L.T.B; FERREIRA, L.; HOEFELMANN, C. P. Valores normativos de aptidão funcional em mulheres de 70 a 79 anos. Revista Brasileira de Cineantropometria e Desempenho Humano, Florianópolis, v.9, n.1, p.28-36, 2007.

BRAVO, G.; GAUTHIER, P.; ROY, P. M.; TESSIER, D.; GAULIN, P.; DUBOIS, M. F.; PELOQUIM, L. The functional fitness assessment battery: Reliability and validity data for elderly women. Journal of Aging and Physical Activity, Champaign, v.2, n.1, p.67-79, 1994.

CAIXETA JUNIOR, A.G.; BARROS, C.L.M. Avaliação das capacidades física e funcional dos idosos participantes dos programas INTEGRAR e AGITA PATOS. Revista do Núcleo Interdisciplinar de Pesquisa e Extensão do UNIPAM, Patos de Minas, n.6, p.96-106, 2009.

CARVALHAIS, A.M.C. O efeito de um programa de treino na aptidão física de idosos de ambos os sexos. 2004. Dissertação (Mestrado em Ciência do Desporto) - Universidade do Porto, Faculdade de Ciências do Desporto e Educação Física, Porto, 2004. CHODZKO-ZAJKO, W.J.; PROCTOR, D.N.; FIATARONE SINGH, M.A.; MINSON, C.T.; NIGG, C.R.; SALEM, G.J.; SKINNER, J.S. American College of Sports Medicine position stand. Exercise and physical activity for older adults. Medicine and Science in Sports and Exercise, Madison, v.41, n.7, p.1510-30, 2009.

CLARK, B.A. Tests for fitness in older adults: AAHPERD Fitness Task Force. JOPERD, Reston, v. 60, n.3, p.66-71, 1989. COELHO, F.G.M.; QUADROS JÚNIOR, A.C.; GOBBI, S. Efeitos do treinamento de dança no nível de aptidão funcional de mulheres de 50 a 80 anos. Revista da Educação Física/UEM, Maringá, v.19, n.3, p.445-51, 2008.

DIAS, R.M.R.; GURJĀO, A.L.D.; MARUCCI, M.F.N. Benefícios do treinamento com pesos para aptidão física de idosos. Acta Fisiátrica, São Paulo, v.13, n.2, p.90-5, 2006.

GOBBI, S. Atividade física para pessoas idosas e recomendações da Organização Mundial de Saúde de 1996. Revista Brasileira de Atividade Física e Saúde, Londrina, v.2, n.2, p.41-9, 1997.

GOBBI, S.; VILLAR, R.; ZAGO, A.S. Bases teórico-práticas do condicionamento físico. Rio de Janeiro: Guanabara Koogan, 2005. IBGE. Séries Estatísticas e Séries Históricas. 2008. Disponível em: <http://www.ibge.gov.br/series_estatisticas/subtema. php?idsubtema $=125>$.

MATSUDO, S.M. Envelhecimento e atividade física. Londrina: Midiograf, 2001.

MATSUDO, S.M.; MATSUDO, V.R.; ARAÚJO, T.; ANDRADE, D.; ANDRADE, E.; OLIVEIRA, L.; BRAGION, G. Nível de atividade física da população do Estado de São Paulo: análise de acordo com o gênero, idade, nível socioeconômico, distribuição geográfica e de conhecimento. Revista Brasileira de Ciência e Movimento, Brasília, v.10, n.4, p.41-50, 2002. MATSUDO, S.M.; MATSUDO, V.K.R.; BARROS NETO, T.L.; ARAÚJO, T.L. Evolução do perfil neuromotor e capacidade funcional de mulheres fisicamente ativas de acordo com a idade cronológica. Revista Brasileira de Medicina do Esporte, São Paulo, v.9, n.6, p.377-87, 2003. 
MIYASIKE-DA-SILVA, V.; VILLAR, R.; ZAGO, A.S.; POLASTRI, P.F.; GOBBI, S. Nível de agilidade em indivíduos entre 42 e 73 anos: Efeitos de um programa de atividades físicas generalizadas de intensidade moderada. Revista Brasileira de Ciências do Esporte, Campinas, v.23, n.3, p.65-79, 2002.

MOBILY, K.E.; MOBILY, P.R. Reliability of the Functional Fitness Test Battery for older adults. Journal of Aging and Physical Activity, Champaign, v.5, n.2, p.150-62, 1997.

JORDÃO NETTO, A. Gerontologia básica. São Paulo: Lemos, 1997.

OSNESS, W.H.; CLARK, B.; HOEGER, W.; RAAB, D.; WISWELL, R. Functional fitness assessment for adults over 60 years: a field based assessment. Reston: The American Alliance for Health, Physical Education, Recreation and Dance, 1990. PAULI, J.R.; SOUZA, L.S.; ZAGO, A.S.; GOBBI, S. Influência de 12 anos de prática de atividade física regular em programa supervisionado para idosos. Revista Brasileira de Cineantropometria e Desempenho Humano, Florianópolis, v.11, n.3, p. 255-60, 2009. SANTOS, S.S.C. Enfermagem geronto-geriátrica: da reflexão à ação cuidativa. João Pessoa: UFPB, 2000.

SEBASTIÃO, É.; HAMANAKA, Á.Y.Y.; GOBBI, L.T.B.; GOBBI, S. Efeitos da prática regular de dança na capacidade funcional de mulheres acima de 50 anos. Revista da Educação Física/UEM, Maringá, v.19, n.2, p.205-14, 2008.

SHIGUEMATSU, R.; OKURA, T. A novel exercise for improving lower-extremity functional fitness in the elderly. Aging Clinical and Experimental Research, Milano, v.18, n.3, p.242-8, 2006.

SILVA, M.P.; SANTOS FILHO, J.A.A.; GOBBI, S. Aptidão funcional de mulheres idosas mediante programa supervisionado de atividades físicas generalizadas ou caminhadas regulares sem supervisão. Revista Brasileira de Atividade Física e Saúde, Londrina, v.11, n.2, p.3-12, 2006.

VITAL, T. M.; PATATAS, J.M.; TAVARES, G.H.; COELHO, A.P.G.M.; COSTA, G.A.; NUNES, J.E.D.; PUGA, G.M. Efeito do treinamento resistido na força muscular e capacidade funcional em idosos ativos. Lecturas Educación Física y Deportes, Buenos Aires, v.16, n.155, p.1, 2011.

YAGUCHI, K.; FURUTANI, M. Na applicability study of the AAHPERD's functional fitness test for elderly American adults to elderly Japanese adults. Environmental Health and Preventive Medicine, Sapporo, v.3, p.130-40, 1998.

ZAGO, A.S.; GOBBI, S. Valores normativos da aptidão funcional de mulheres de 60 a 70 anos. Revista Brasileira de Ciência e Movimento, Brasília, v.11, n.2, p.77-86, 2003.

ZAGO, A.S.; POLASTRI, P.F.; VILLAR, R.; SILVA, V.M.; GOBBI, S. Efeito de um programa geral de atividade física de intensidade moderada sobre os níveis de resistência de força em pessoas da terceira idade. Revista Brasileira de Atividade Física e Saúde, Londrina, v.5, n.3, p.42-51, 2000.

\begin{tabular}{|c|c|}
\hline $\begin{array}{r}\text { ENDEREÇO } \\
\text { Deisy Terumi Ueno } \\
\text { Av. 46 A, 1175 - apto. } 6 \\
\text { 13506-600 - Rio Claro - SPR - BRASIL } \\
\text { e-mail: terumiueno@uol.com.br }\end{array}$ & $\begin{array}{l}\text { Recebido para publicação: 07/ 01/2011 } \\
\text { 1a. Revisão: 13/06/2011 } \\
\text { 2a. Revisão: 18/01/2012 } \\
\text { Aceito: 14/ 02/2012 }\end{array}$ \\
\hline
\end{tabular}

